$\mathrm{PET} / \mathrm{CT}$ examination was performed and its results were evaluated by both qualitative scoring, on a 5-grade scale from 0 (no uptake) to 4 (clearly higher than liver uptake), and analysis of the standardised uptake value (SUV max and mean) in a $\mathrm{ROI}$ placed on the deltoid, byceps, gluteus and quadriceps muscles. A ROI of the joints and bursae was also designed and the SUV calculated. The muscle ROls were positioned distant from the corresponding joints to avoid interference from possible articular or periarticular uptake. Demographic, clinical and laboratory data were collected.

Results: PMR patients showed an uptake higher than that of controls in the deltoid $(p=0.0004)$, gluteus $(p=0.015)$, and quadriceps $(p=0.009)$, but not in the biceps $(p=0.06)$ muscles. The semiquantitative SUV evaluation was consistent for the deltoid (SUV mean, $p=0.047$ ) and gluteus (SUV mean, $p=0.01$; SUV max, $\mathrm{p}=0.006$ ) muscles. There was no correlation between muscle uptake and that of the adjoining joint. Similarly, no correlation was found between muscle uptake and demographic, clinical and laboratory (CRP, ESR) findings.

Conclusions: PMR show muscular inflammation at FDG-PET/CT, which does not derive from the nearby joints or periarticular tissues. A clearly defined myositis is not probable, because creatine kinase concentrations are normal in PMR. However, muscle inflammation may contribute to the global inflammatory burden and symptoms of patients with PMR.

Disclosure of Interest: None declared

DOI: 10.1136/annrheumdis-2018-eular.3219

\section{THU0455 AGE IS MORE CLOSELY ASSOCIATED WITH POSITIVE TEMPORAL ARTERY BIOPSY OVER BIOPSY LENGTH IN LARGE VETERANS ADMINISTRATION DATABASE STUDY}

M.B. Morcos ${ }^{1}$, S. Chung ${ }^{1}$, B. $\mathrm{Ng}^{1,2} .{ }^{1}$ Rheumatology, University of Washington;

${ }^{2}$ Rheumatology, Veterans Administration, Seattle, USA

Background: Giant cell arteritis (GCA) is a granulomatous vasculitis with a predilection for older women. While American College of Rheumatology (ACR) diag nostic guidelines exist, temporal artery biopsy (TAB) remains an essential tool often used independently to substantiate long-term steroid use and/or immunosuppression. No consensus exists regarding the ideal length of biopsy to optimise pathological yield. The influence of demographic factors such as age, race, and gender on the yield of biopsies is not defined.

Objectives: To determine if length of TAB and patient's race, gender, or age at biopsy influenced TAB results using the Veteran's Administration (VA) national database.

Methods: Patients with a procedure code for TAB between 1999-2017 were queried through the VA national database. The biopsy length and result (positive, negative, or indeterminate) were recorded. Demographic information including subject age, gender, and race was also extracted. Logistic regression models were run using Stata to identify independent determinants of a positive TAB.

Results: 2136 biopsies have been randomly reviewed to date. The average length of TAB was $12.15 \mathrm{~mm}$. TAB results were $9 \%$ positive, $89 \%$ negative, and $2 \%$ indeterminate. There was no statistically significant association between biopsy length and a positive result; however, when compared to the reference group ( $>10 \mathrm{~mm}-<15 \mathrm{~mm}$ ), the odds ratio for positive results increased with specimen length $>20 \mathrm{~mm}$ to $30 \mathrm{~mm}$ in length. Conversely, there was a trend towards negative biopsy results for samples $<10 \mathrm{~mm}$ in length when compared to the reference length group. There was no correlation between TAB result and race or gender; however, age correlated with a positive biopsy which was statistically significant. None of the subjects $<50$ years of age had a positive TAB. Younger age groups (age categories 50-59 and 60-69) were less likely to have a positive TAB (OR of $0.32 \mathrm{Cl} 0.17-0.61$ and OR $0.53 \mathrm{Cl} 0.38-0.74$, respectively) when compared to the reference age group (age 70-79). Conversely, older populations aged 90 and above demonstrated a statistically significant increased likelihood of a positive biopsy result with an OR of $2.25, \mathrm{Cl} 1.05-4.85$.

Conclusions: The incidence of positive TAB among patients with suspected temporal arteritis in the VA national database was surprisingly low. The effect of other factors, such as the referring specialty or pre-biopsy steroid use, on TAB result may be insightful in understanding the low yield. Age remains a helpful tool given its association with increased likelihood of a positive biopsy, and the decision to pursue TAB in patients $<50$ years old should be approached judiciously. Length of biopsy was not associated with a positive result; however, the trends we note suggest a biopsy between $10 \mathrm{~mm}$ and $25 \mathrm{~mm}$ could optimise the yield. Gender and race were not helpful predictors for biopsy yield in this population.

Disclosure of Interest: None declared

DOI: 10.1136/annrheumdis-2018-eular.1437

\section{THU0456 \\ THE CLINICO-HISTOPATHOLOGICAL ALGORITHM FOR DIFFERENTIAL DIAGNOSIS OF BUERGER'S DISEASE, TAKAYASU ARTERITIS AND ATHEROSCLEROTIC OCCLUSIVE DISEASE}

M. Bagriy ${ }^{1}$, O. Zimba2 ${ }^{1}{ }^{1}$ Department of Pathomorphology and Legal Medicine, Ivano-Frankivsk National Medical University, Ivano-Frankivsk; ${ }^{2}$ Department of Internal Medicine, Danylo Halytsky Lviv National Medical University, Lviv, Ukraine

Background: Arterial occlusive disease is a significant cause of the disability due to lower limbs amputations. In addition to atherosclerosis, systemic vasculitides can present with progressive critical limb ischemia and could be misdiagnosed as atherosclerotic occlusive disease.

Objectives: To investigate the pathological findings in vessels of the lower limbs amputated due to Takayasu arteritis (TAK), Buerger's disease (BD) in comparison to atherosclerotic occlusive disease (AOD) and diabetic angiopathy (DA). Additionally, to develop an algorithm for clinico-histopathological differential diagnosis. Methods: The specimens of vessels segments were obtained from the nine anatomical levels of amputated lower extremities in 132 patients, of which 30 were with TAK, 42 with BD, 30 with AOD and 30 with DA.

Results: In cases of $\mathrm{BD}$, the exudative-productive endo-mesoarteritis with intimal hyperplasia from stenosis to complete obliteration have been found in the lower leg and foot arteries. No morphological abnormalities were observed in proximal arteries above knee. The pathologic hallmark of BD was panphlebitis with intimal hyperplasia, most often in anterior and posterior tibial veins, vena dorsalis pedis and superficial vein. The most remarkable finding in cases of TAK was mesoarteritis and luminal narrowing due to the reactive intimal hyperplasia in proximal arterial segments. In approximately half of the cases, TAK was not diagnosed clinically and identified only based on the pathological examination. The most common incorrect clinical diagnosis was atherosclerotic occlusive disease. In seven cases of TAK, concomitant premature atherosclerosis of varying severity was observed. Interestingly, in our series of cases of TAK, males were most frequently affected. The correct clinical diagnosis of TAK was made in all females' cases. It seems to be a trend to miss the clinical diagnosis of TAK in men. In cases of $A O D$, pathological examination revealed stenotic atherosclerotic plaques occurred most prominently in the tunica intima of femoral and popliteal arteries. The most common morphologic features in cases of DA were media calcinosis and fibrous lipid plaques both distal and proximal arterial segments. The algorithm for clinico-histopathological differential diagnosis of TAK, BD, AOD and DA consists of the following: a) the best diagnostic performance can be obtained with four-five specimens of the magistral vessels from the proximal and distal parts of the amputated limb and the superficial vein at the level of the upper third of the shin; b) using routine and special staining techniques ( $\mathrm{H}$ and $\mathrm{E}$, Masson's trichrome stain for collagen fibres, Hart's elastin stain); c) analysis of the demographic and clinical data; $d$ ) interpretation of pathologic changes in vessels of the lower limbs; e) making a pathologic diagnosis.

Conclusions: The current findings could contribute to the improving the differential diagnosis of Takayasu arteritis, Buerger's disease, atherosclerotic occlusive disease and diabetic angiopathy.

Disclosure of Interest: None declared

DOI: 10.1136/annrheumdis-2018-eular.7500

\section{THU0457 INTERLEUKIN-6 EXPRESSION IN INFLAMED AND NON- INFLAMED TEMPORAL ARTERIES FROM PATIENTS} WITH GIANT CELL ARTERITIS

N. Pipitone $^{1}$, F. Muratore ${ }^{1}$, I. Tamagnini ${ }^{2}$, A. Cavazza ${ }^{2}$, L. Cimino ${ }^{3}$, L. Boiardi ${ }^{1}$, G. Restuccia ${ }^{1}$, M. Bonacini ${ }^{4}$, S. Croci ${ }^{4}$, C. Salvarani ${ }^{1}{ }^{1}$ Rheumatology Unit, ${ }^{2}$ Pathology Unit, ${ }^{3}$ Ophthalmology Unit, ${ }^{4}$ Clinical Immunology, Allergy and Advanced Biotechnologies Unit, Azienda USL-IRCCS di Reggio Emilia, Reggio Emilia, Italy

Objectives: To evaluate if interleukin-6 (IL-6) expression in the temporal artery biopsy (TAB) specimens may differentiate patients with giant cell arteritis (GCA) from those without.

Methods: 63 consecutive formalin-fixed, paraffin-embedded (FFPE) TABs performed between 2009 and 2012 from 32 patients with transmural biopsy-proven GCA, 8 patients with biopsy-negative GCA and 23 controls were retrieved. Demographic, clinical, and laboratory data at presentation and at each follow-up visit were collected. A pathologist reviewed all TABs. Immunohistochemistry was performed on $4 \mu \mathrm{m}$ FFPE tissue sections with a 1:400 dilution of rabbit polyclonal anti-human IL-6 antibody (NOVUS Biologicals Littleton, Co.) for 60' at $37^{\circ}$. Slides of TAB specimens were independently assessed by five readers. IL- 6 expression was graded as 0 (absent), 1 (mild), 2 (moderate) and 3 (marked). Inter-reader differences were resolved by consensus. Anti-IL6 staining was considered positive if staining was grade 2 or 3 , since grade 1 was faint, sometimes difficult to differentiate from background, and showed the least degree of agreement between readers. 